\title{
Remote Mobile Environmental Monitoring System based on Socket Lianzhen Huang ${ }^{1, a}$, Lin Zhao ${ }^{2, b^{*}}$, Qinggang Tu ${ }^{3, \mathrm{c}}$, Jianbo Wu ${ }^{4, d}$, Heming Zhao ${ }^{5, e}$ and Jinzhin Ren ${ }^{6, f}$
}

\author{
School of Southwest Minzu University, Chengdu, Sichuan, 610041, China \\ a2285317264@qq.com, b277400572@qq.com, c1109768128@qq.com, ${ }^{\mathrm{d}} 1104093585 @ q q . c o m$, \\ e1129914574@qq.com, ${ }^{f} 1442465025 @ q q . c o m$ \\ *Corresponding author e-mail: 277400572@qq.com
}

\begin{abstract}
Keywords: Remote environmental monitoring; Socket multithreading communication; Data dynamic interaction; Real-time processing
\end{abstract}

\begin{abstract}
This article designed and implemented a remote mobile environmental monitoring system based on Socket communication technology. The system consists of clients, a server, and remote terminals. The interaction between the client and the remote terminal of the system is based on the server acting as an intermediate medium for transmission. Not only the system can move freely, without restrictions to the use of places, but also can realize the function of remote control and network data transmission between the client and the remote terminal. At the same time, the data interaction of the whole system has real-time dynamic characteristics. The experimental results turn out that the system's rapid response and stable performance achieve the remote control requirements. We believe that this system will have strong practicalities in remote environmental monitoring.
\end{abstract}

\section{Introduction}

Traditional environmental monitoring is the collection of manual data by physical means, chemical means, and biological means [1]. Traditional environmental monitoring aiming at collecting environmental information entails a lot of manpower and material resources, also is limited by time and area. What is more, it cannot obtain real-time data. After data collection, it also requires a long data processing process. It can be seen that traditional methods of environmental information collection no longer meet the actual needs of current environmental monitoring. For these problems, it is of great significance to design a dynamic, real-time and automatic environmental monitoring system through the advanced computers, networks, communications and other technologies, and so on [2].

Socket which uses standard UNIX file descriptors to communicate with other programs is commonly called "socket" [3]. There are two kinds of standard sockets, one is Stream Socket with reliable, connection-oriented TCP service; the other is Datagram Socket without connectional service. We use the streaming socket of TCP/IP (Transmission Control Protocol/Internet Protocol) protocol to communicate between service programs. In addition, to meet the needs of the system for rapid response and real-time processing of dynamic interactive data, multithread as a powerful technical support [4] provides ways for the server to solve multiple clients request for concurrent connection problems. Multithreading has many advantages, such as high speed, less resources, data sharing and so on [5], which meet the design requirements of dynamic real-time system.

In a word, multithread and Socket promote the communication between application programs, respectively, the client and server programming. So we use them to design a remote mobile environmental monitoring system.

\section{The Overall Structure of the System}

Remote mobile environmental monitoring system in this paper mainly includes three parts: the client, the server and the remote terminal. The workflow of the system is roughly: 1) Web interface of the client provides users with remote control and data display interfaces. The browser requests to connect to the server using HTTP (Hypertext Transfer Protocol) protocol. 2) After successful connection, the server returns the data result to the client. The server also processes the remotely 
transmitted data, which serves as a bridge for providing data interaction between the client and the remote end of the system. 3) The remote terminal connects to the server through Socket communication, at the same time it also accepts instructions from the server and then performs the corresponding actions. In a short word, whether the browser is or the remote end, their communication with the server or network data transmission is performed in the Internet. As long as both parties of the communication obey the same protocol, they can communicate and transmit information. The structure of the system is shown in Fig. 1,

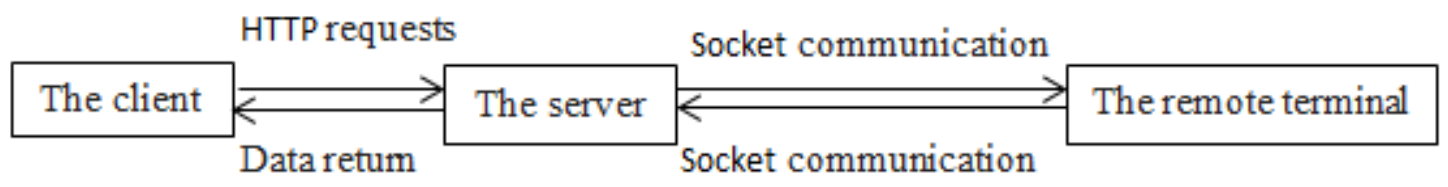

Figure 1 Overall system block diagram

\section{Hard ware Structure Design}

The hardware module mainly includes a mobile robot car and other external equipment, which is composed of remote terminal system. The other equipment here is several different types of sensors, a camera, a networking module, lights, fans and pumps, which are all packaged on the robot car so that the car can capture remote environmental information. The sensors mentioned in this paper are DHT11 digital temperature and humidity sensor, MQ-135 air quality sensor, and SR501-HC human infrared sensor.

The remote monitoring system's workflows are: first, the core control module detects the working status of the robot car, lights, fans, and pumps. The sensors and camera collect the environmental monitoring data of the remote terminal, after the acquisition is completed, the core control module transmit the information to the server through the networking module. The server then receives and processes it immediately. Therefore, using the car as the carrier of the remote monitoring system, the advantage is that it can realize autonomous movement, and at the same time carry lighting, alarm equipment, watering, cooling and acquisition of remote pictures. The structure of the remote system is shown in Fig. 2,

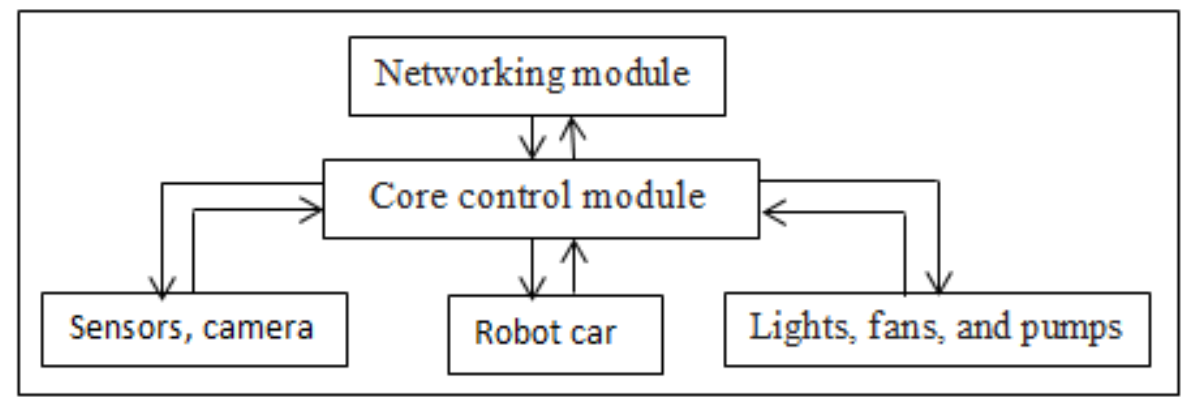

Figure 2 The structure of the remote system

\section{Software Design of the System}

Data transmission form in the network. To achieve the purpose of controlling a remote device and acquiring remote data, a method must be used to send an instruction to the remote end, and then the remote end performs the corresponding action and returns information to the client. Therefore, transmission over the Internet is a good choice and it is the mainstream communication method in the modern era.

The process of data transmission in the network can be summarized as: first, the data and HTTP headers form a TCP segment, and then the TCP segment add the IP header constitutes an IP segment. The IP segment add the Ethernet header and tail, the data can be transmitted in the Internet. 
Data is transmitted from the client to the server, where returns data to the client, actually this is the encapsulation and unsealing process of the data transmitted. These two processes form the form of sending and receiving data on both sides of the communication, as shown in Fig. 3,

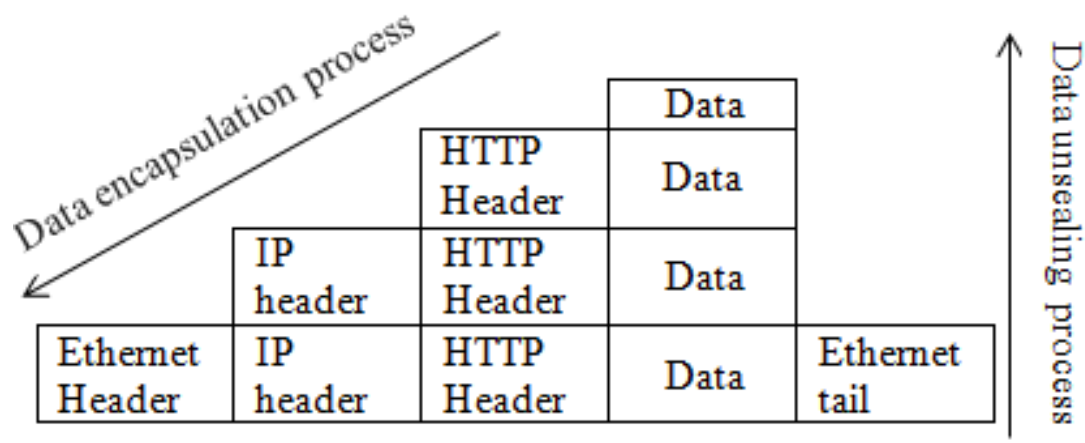

Figure 3 The process of data transmission in the Internet

Communicate between the remote-side and the server-side programs. At runtime, start the server and open the remote controller first, the server will call the Socket() function, call the Bind() function to bind the Socket and the address, and use the Listen() function to start monitoring. After receiving a connection request from the remote terminal, the server establishes a virtual communication link with it, and finally two communication terminal devices call Recv() and Send() functions to perform two-way communication. In this system design, the remote monitoring system needs to complete two tasks. One task is to receive commands from the web client. Another task is to execute the command, and then the monitored data is returned to the client. The communication structure is shown in Fig. 4,

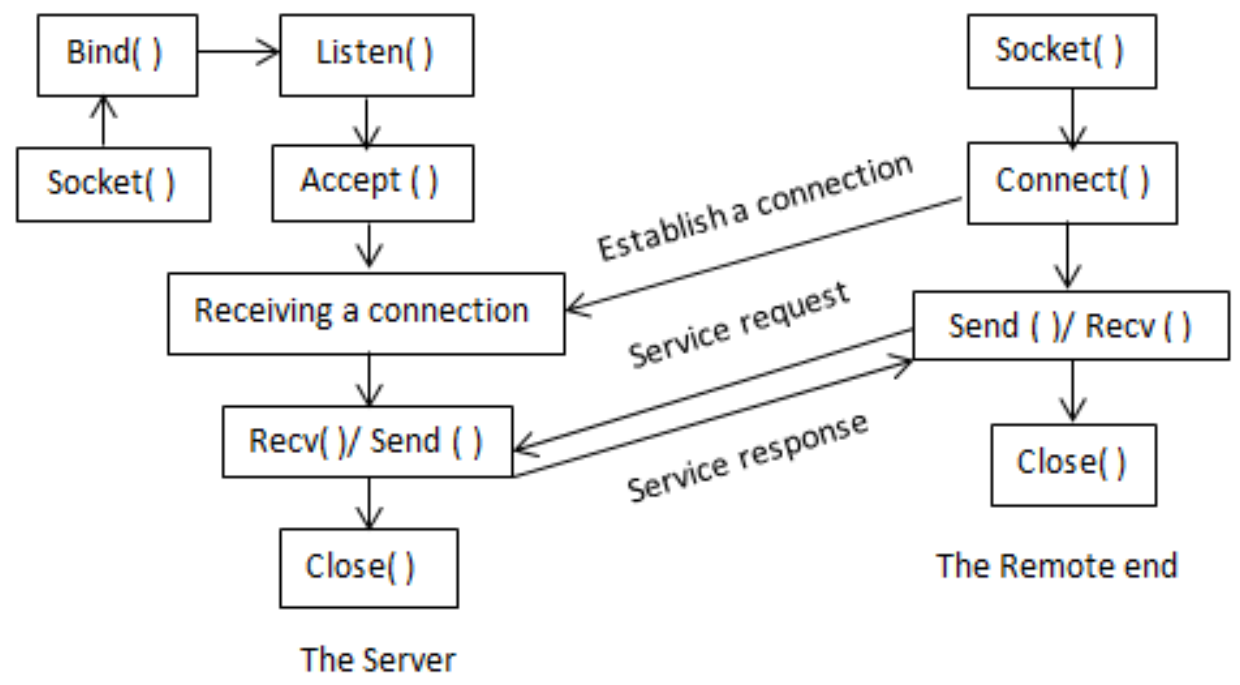

Figure 4 Socket communication between the server and the remote end

In this system design, we adopt the multithreaded mode of Socket communication technology. When there are multiple terminals requesting connection at the same time, the server-side main control thread starts the sub-thread in a separate state to handle service requests from the remote end. Experiment shows that the model greatly improves the server's concurrent [6] processing capabilities, as shown in Fig. 5, 


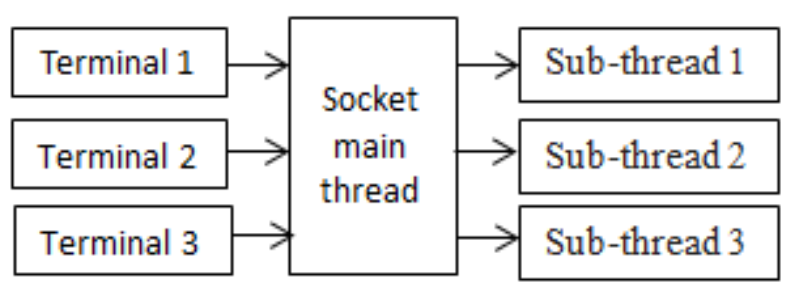

Figure 5 Socket's multi-threaded model

Logging and data information storage when the system is working. Server-side configuration files used in this system, which recording the two-way communication between the connection side and the server side, the connection refers to the client or the remote end. The data collected by the network is stored in the background SQL (Structured Query Language) database [7] of the server for users to query at any time. The database is easy to cross-platform, has a good portability, using the database statement can easily add, delete, modify, query and other operations. Therefore, the user can conveniently query the environmental data uploaded by the network through an interactive friendly client interface.

\section{System Overall Test}

The user controls and manages the remote embedded devices by the web interface of the browser. In the web page area, the working status of each controlled object and the data information collected by the network are displayed in real time. This system finally achieved the design goals of remote control of robotic car, lights, fans, pumps, and access to remote environmental temperature and humidity, gas concentrations, and pictures to meet functional requirements. The control of each controlled object is as follows.

The user can give orders to "forward", "left", "right", "back", and "stop" to the robot car. When it is necessary to control the movement of the car, click the control button, the command information will be added to the TCP/IP data transfer protocol with an HTTP request and uploaded to the server through the Internet. The server platform sends the relevant command information to the robot for analysis, and the car performs the related operations in a short time. The control interface is shown in Fig. 6,
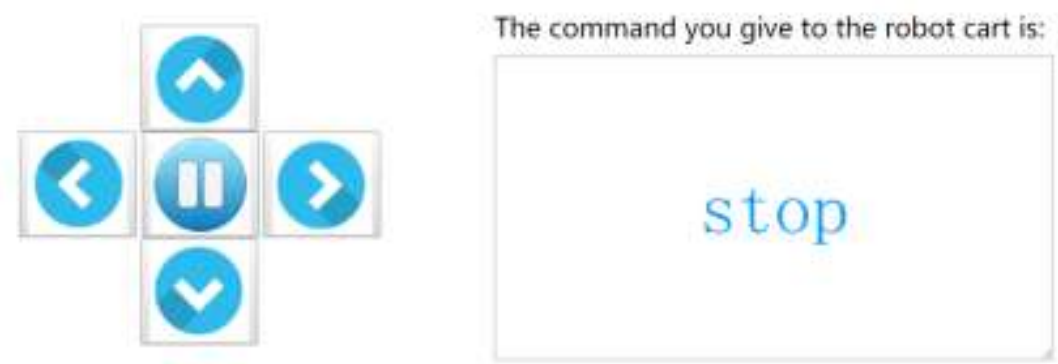

Figure 6 The interface of control robot car

The controlled object light, fan, and water pump are used to simulate the remote environment. The light is used for lighting, the fan is used to cool the temperature, and the water pump is used to give rainfall to the remote environment. Click the corresponding button, the information will be transmitted to the networked core control module, and the core control module will control controlled objects through the I/O (Input or Output) ports. As shown in Fig. 7,

\section{Turn on the fan Turn off the fan}

$$
\text { Turn on the light Turn off the light }
$$

Figure 7 The interface of control remote simulation objects 
After clicking the "Click the button to query remote environmental information" button, the core control module uploads the data collected by the sensor to the server platform in Socket multi-thread communication mode. The server stores the data, and then reads the data from the back-end database. The data of the user interface is updated, but the page does not jump [8], as shown in Fig. 8,

Click the button to query remote environmental information

Information query succeeded!

Remote temperature update time:Sun Apr 15 16:05:32 2018

Temperature is: $22\left({ }^{\circ} \mathrm{C}\right)$ Humidity is: $17 \%(\mathrm{RH})$ Gas concentration is: $30(\mathrm{PPM})$

Figure 8 Remote environmental weather information

The web page also shows the environmental images collected by the remote camera at the most recent moment. The image contains the remote-end screen information that the user wants to know. The image is uploaded every ten seconds and updated on the user interface in real time, as shown in Fig. 9. The text on the right side of the picture describes the time when the remote picture was uploaded.

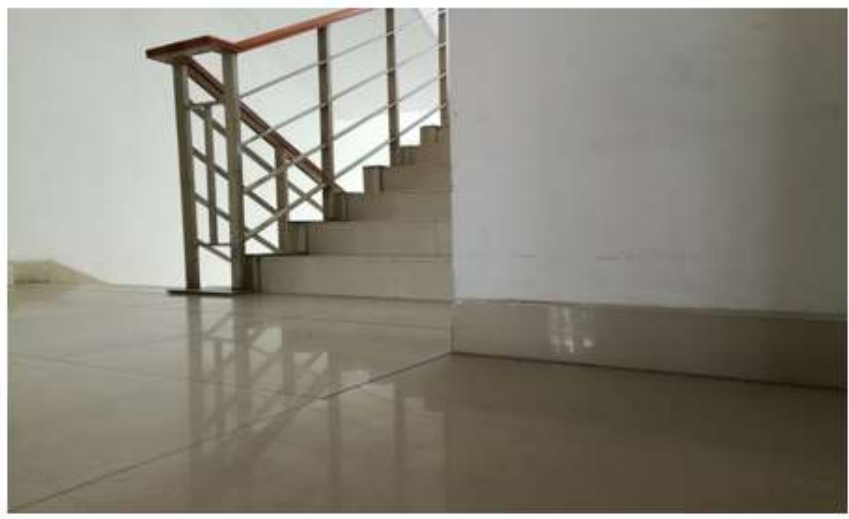

The picture on the left is taken by a remote camera. The camera will upload an image every 10 seconds, and the user interface will be displayed in real time.

The upload time on the left picture is: 2018-04-21 14:27:55

Figure 9 Photo taken by a remote camera

\section{Summary}

Under the TCP/IP network data transmission protocol based on the Socket multithreading model, we completed the data collection of remote environment, network data transmission, server processing data, and the system design of the client interface. The environmental monitoring system realizes the function of dynamic data interaction between client and server, remote terminal and server. The system overcomes the shortcomings of traditional environmental monitoring that are limited by time and area. It can be started and stopped at any time. After testing, the system can be moved flexibly with stable and strong real-time performances. We believe the system has a strong practicality.

\section{Acknowledgement}

This article supported by Research and Reform Project on Education and Teaching of Southwest Minzu University: 2018, Development of a Multi-Functional Platform for Sensor Experiment Teaching; 2017, Research on Sensor Experiment Teaching Reform. 


\section{References}

[1] L.D. Chen, J.H. Feng and B.G. Xu. Journal of Tongji University. Vol. 38 (2010) No.11, p1659 (In Chinese)

[2] B. Xue, N. Yan. Urban Construction Theory Research: Electronic edition. (2013) No.15 (In Chinese)

[3] R.H. Zhang. Science and Technology Information: Science Teaching and Research. (2008) No.9, p74 (In Chinese)

[4] Y.G. Zhang, C.C. Liu, W. Liu and Z.F. He. Control Engineering. Vol. 13 (2006) No.2, p175 (In Chinese)

[5] J.B. Song: Linux Network Programming(Tsinghua University Press, China 2014), p425 (In Chinese)

[6] S. Li. Computer Knowledge and Technology. (2014) No.28, p6764 (In Chinese)

[7] Y. W, J. Y and L. G. Electronic technology. Vol. 38 (2011) No.7, p12 (In Chinese)

[8] H. Y. Computer Knowledge and Technology. Vol. 5 (2009) No.18, p4782 (In Chinese)

[9] Peng L, Xiaoping Z. Social Stratification and Cooperative Behavior in Spatial Prisoners' Dilemma Games [J]. PLOS ONE, 2015, 10(7): e0131005. 